\title{
EL SISTEMA ELECTORAL ITALIANO Y SU REFORMA: EL DESAFÍO DE LA CONSOLIDACIÓN
}

\author{
FRANCESCO CLEMENTI \\ Profesor de Derecho Publico Comparado \\ Universidad de Perugia
}

TRC, núm. 45, 2020, pp. 185-206

ISSN 1139-5583

\section{SUMARIO}

I. Introducción. II. El sistema electoral italiano en la primera fase republicana (1948-1993). III. El sistema electoral italiano en la segunda fase republicana (1994-2020): veintiséis años intensos, entre ya y todavía no. III. 1. La ley Mattarella (1994-2005): un sistema electoral mixto. III. 2. La ley Calderoli (20052016): un proporcional con premio mayoritario (2006-2016). IV. ¿Una tercera fase? Gobiernos populistas y sistemas electorales. (2016-2018). V. ¿Hacia un nuevo sistema electoral? Las propuestas más recientes (2018-2020). VI. Conclusión. El sistema electoral italiano y el desafío de la consolidación.

\section{INTRODUCCIÓN}

Durante las últimas seis legislaturas italianas (1994-2020) —las posteriores a la llamada «Primera República» ${ }^{1}$ — se han celebrado siete elecciones generales y se han constituido dieciséis gobiernos ${ }^{2}$. En ese mismo período se han convocado

1 Este término periodístico se refiere al sistema político de la República Italiana vigente entre 1948 y 1994, explosionado con el fin de la Guerra Fría entre el Este y el Oeste del mundo, y, en el caso italiano, principalmente gracias a tres elementos: el referéndum abrogatorio del 9 de junio de 1991 en favor de una preferencia única para la elección de la Cámara de Diputados, la investigación judicial sobre el sistema de sobornos recibidos por los políticos (caso «Mani pulite»), que comenzó en febrero de 1992, en la que participaron muchos exponentes de todos los partidos principales y que puso de manifiesto el fenómeno conocido entonces como «Tangentopoli»; por último, pero no por ello menos importante, el referéndum del 18 de abril de 1993 en el que se reformó la ley electoral del Senado de la República hacia un sistema mayoritario.

2 Legislatura XII (15 de abril de 1994 - 16 de febrero de 1996, con los gobiernos: Gobierno Dini y Gobierno Berlusconi I); XIII Legislatura (9 de mayo de 1996 - 9 de marzo de 2001, con los gobiernos: Gobierno Prodi, Gobierno D’Alema, Gobierno D’Alema II y Gobierno Amato II); XIV Legislatura (30 de 
cuatro referendos de revisión constitucional ${ }^{3}$, se han aprobado cuatro leyes electorales para las elecciones generales ${ }^{4}$ y se han dictado tres sentencias del Tribunal Constitucional sobre la ley electoral para estas elecciones. ${ }^{5}$

Esta intensa actividad en el frente político-institucional se ha visto acompañada por una vitalidad muy similar del sistema de los partidos políticos. En efecto, se ha producido, por una parte, una fuerte remodelación del sistema de partidos, con la desaparición de los partidos clásicos y el nacimiento de nuevas formaciones políticas que han entrado en el Parlamento, y, por otra, un profundo cambio de la clase político-parlamentaria. De los partidos políticos presentes en el Parlamento antes de la XII Legislatura (1994), sólo queda hoy en día la formación política de la Liga (que, sin embargo, ya no se califica como Liga del Norte); cabe apuntar como dato anecdótico que el decano de los parlamentarios electos en la composición actual tiene sesenta y dos años.

En resumen, los últimos treinta años de la vida política e institucional italiana han sido ciertamente intensos y han sacudido con creces la Constitución material, es decir, la aplicación concreta por parte de las fuerzas políticas de los principios, normas y derechos enunciados en la Constitución formal ${ }^{6}$.

mayo de 2001 - 27 de abril de 2006, con los gobiernos: Gobierno de Berlusconi II y Gobierno de Berlusconi III); XV Legislatura (del 28 de abril de 2006 al 6 de febrero de 2008, con el Gobierno Prodi II); XVI Legislatura (del 29 de abril de 2008 al 23 de diciembre de 2012, con los gobiernos: Gobierno Berlusconi IV y Gobierno Monti); XVII Legislatura (del 15/03/2013 al 22/03/2018, con los gobiernos: Gobierno Letta, Gobierno Renzi y Gobierno Gentiloni); XVIII Legislatura (el Gobierno Conte I, del 1 de junio de 2018 al 5 de septiembre de 2019, y luego por el Gobierno Conte II, en el cargo desde el 5 de septiembre de 2019 hasta ahora).

3 El último será el 29 de marzo de 2020 para una reducción general del número de diputados de 945 a 600 componentes. Vid. F. CLEMENTI, «Sulla proposta costituzionale di riduzione del numero dei parlamentari: non sempre «less is more»», en Osservatorio sulle Fonti, $\mathrm{n}^{\circ} 2,2019$.

4 En particular, se hace referencia a: la llamada ley electoral llamada Mattarella, bautizada más tarde por Giovanni Sartori, a causa de su primer signatario, con el «latinorum» de Mattarellum (leyes del 4 de agosto de 1993 n $^{\circ} 276$ y n 277); la ley electoral de Calderoli, bautizada — de nuevo por Giovanni Sartoricomo la llamada Porcellum (ley del 21 de diciembre de 2005 n 270); la llamada ley electoral. Italicum, para el sistema electoral único de la Cámara de Diputados, ya que se imaginaba que el resultado del referéndum del 4 de diciembre de 2016 haría que el Senado de la República no fuera elegido directamente, bautizado así por su principal patrocinador Matteo Renzi, entonces Presidente del Consejo, (ley del 6 de mayo de 2015, $\mathrm{n}^{\circ}$ 52); para llegar, finalmente, después de altibajos de los que se dirá, al actual llamado Rosatellum bis, bautizado así por su autor principal Ettore Rosato, entonces líder de la Cámara de Diputados del grupo parlamentario del Partido Demócrata (ley del 3 de noviembre de 2017, n 165).

5 La última fue la sentencia $\mathrm{n}^{\circ} 10$ de 2020 por la que el Tribunal Constitucional declaró inadmisible la solicitud de referéndum popular sobre la «Abolición del método proporcional en la asignación de escaños en circunscripciones pluralistas en el sistema electoral de la Cámara de Diputados y el Senado de la República», presentada por los Consejos Regionales de Véneto, Piamonte, Lombardía, Friul-Venecia Julia, Cerdeña, Abruzos, Basilicata y Liguria.

6 Para una noción completa del concepto de «Constitución en el sentido material», Vid. C. MorTATI, La Costituzione in senso materiale, Milano, Giuffré, 1998; amplius, vid. F. LANCHESTER (Ed.), Costantino Mortati costituzionalista calabrese, Napoli, Jovene, 1989; M. Galizia - P. Grossi (Ed.), Il pensiero giuridico di Costantino Mortati, Milano, Giuffré, 1990; M. Fioravanti, La scienza del diritto pubblico. Dottrine dello Stato e della Costituzione tra Otto e Novecento, 2 voll., Milano, Giuffré, 2001. 
En este contexto, el sistema electoral y sus continuas modificaciones con nuevas leyes electorales — como ya demostró Duverger en sus estudios de principios de los años cincuenta - ha influido fuertemente en la forma de gobierno, marcando, por el factor decisivo de condicionamiento que representa el sistema de partidos, su dinámica, tanto directa como indirectamente.

Sin embargo, el rápido cambio de los diferentes sistemas electorales en los últimos años no ha estado acompañado de una reforma constitucional —a pesar de tres décadas de intentos_- por lo que ha habido una asimetría en el funcionamiento de los nuevos sistemas electorales que se iban adoptando gradualmente, que los hacía sustancialmente incompletos, incoherentes $y$, en definitiva, frágiles.

Por lo tanto, frente a una inmutabilidad del sistema constitucional, esta continua mutabilidad en el sistema electoral no solo ha provocado que todo el sistema político-institucional haya sido muy débil y víctima de una búsqueda continua y frenética del mejor sistema electoral adecuado para una nueva era de representación política, sino que también lo ha degradado en su función a los ojos de la ciudadanía, ya que parecía una herramienta claramente ineficiente con respecto a las necesidades del sistema constitucional. Por otro lado, solo podría ser así, considerando que se intentó introducir un nuevo sistema electoral en un texto constitucional que, de hecho, a pesar de los intentos, ha permanecido casi sin cambios desde 1948 en los instrumentos propios de la forma de gobierno.

De esta forma, siguiendo un continuo instinto peligroso y autodestructivo y a pesar de las diversas propuestas hechas a lo largo de los años por las fuerzas políticas para introducir un cambio político-constitucional gracias a la invocación y a los tentativos periódica de «reformas», nunca ha sido posible estabilizar por un tiempo razonable un sistema electoral, precisamente porque el sistema político era inestable y la estructura político-institucional, casi inalterable.

Teniendo en cuenta todo esto, a pesar de la ausencia de cesuras constitucionales formales, la revolución político-institucional que tuvo lugar entre 1989 y 1993 obligó a la doctrina a dividir los setenta años de vida republicana en dos períodos: la llamada Primera República (1948-1994) y la llamada Segunda República (1994-2020), expresiva de un primer y un segundo sistema de partidos políticos, así como de diferentes y numerosas leyes electorales.

En las presentes notas se pondrán de relieve las líneas de persistencia y cambio del derecho electoral en consideración de las transformaciones producidas en el marco de la llamada Segunda República y de su nuevo sistema de partidos políticos, tratando de subrayar las dificultades de su consolidación, también a la luz de los factores extralegales y meta-legales que afectan al dinamismo de las fuerzas políticas dentro de las estructuras institucionales. ${ }^{7}$

7 G. Amato - F. Clementi, Forme di Stato e forme di Governo, Bologna, Il Mulino, 2012. 


\section{EL SISTEMA ELECTORAL ITALIANO EN LA PRIMERA FASE REPUBLICANA (1948-1994)}

Ya en el umbral del nacimiento de la República Italiana había plena conciencia de la naturaleza y de las múltiples funciones que el sistema electoral desempeña en un sistema.

En efecto, la «Comisión para la elaboración de la ley política electoral para la Asamblea Constituyente», tuvo que definir en el informe final de sus trabajos con respecto al procedimiento de elección de la Asamblea que se había de constituir que «ningún procedimiento electoral es perfecto; todos están vinculados a las condiciones políticas y sociales de un pueblo en un período histórico determinado y los resultados dependen no tanto de los medios instrumentales, sino de la conciencia y la madurez política de quienes los utilizan». ${ }^{8}$

Con ese planteamiento se aprobó la nueva ley electoral para el nacimiento de la Asamblea Constituyente (Decreto Legislativo n. 74 del 10 de marzo de 1946) que estableció un sistema proporcional mediante la votación de listas de candidatos en libre competencia, y que se convertiría entonces en el modelo para la definición del subsiguiente sistema electoral de la Cámara y del Senado, tan pronto como la Constitución fuera aprobada definitivamente.

De forma concreta, el sistema electoral que caracterizó gran parte de la historia republicana italiana fue establecido, para el Senado, en virtud de la Ley ${ }^{\circ}$ 29 de 6 de febrero de 1948, y, para la Cámara, por la Ley n 1058 de 7 de octubre de 1947, que introdujo un sistema electoral proporcional (en el seno de circunscripciones pluri-provinciales concebidas como secciones del colegio nacional único) a las listas en competencia, con la posibilidad de expresar tres o cuatro preferencias, según el tamaño de las circunscripciones.

En esta primera fase de la República, la estabilización definitiva del sistema electoral nacional en una dirección totalmente proporcional ocurre por dos razones.

Por una parte, por razones de política interna, ya que el partido principal, la Democrazia Cristiana, siendo el partido que siempre ha tenido el mayor número de votos, con el sistema electoral proporcional logró ser el principal partido del gobierno, certificando así su papel central en todo el sistema político; un hecho tan claro que intentó marcar su centralidad a través de la introducción de una ley —llamada «Legge Truffa»— que daba un premio mayoritario a aquellos que

8 La referencia se encuentra en la página 18 del informe, que puede leerse hoy en el sitio web institucional de la Cámara de Diputados. En cualquier caso, vea todos los actos en: Ministero per la Costituente (Ed.), Atti della Commissione per la elaborazione della legge elettorale politica per l'Assemblea costituente, Roma, Tip. U.E.S.I.S.A., 1946. Vid. G. D’Alessio (Ed.), Alle origini della Costituzione italiana: i lavori preparatori della Commissione per studi attinenti alla riorganizzazione dello Stato (1945-1946), Bologna, il Mulino, 1979. 
superaban el $50 \%$ de los votos. ${ }^{9}$ Cuando esta tentativa fracasó, se propuso volver al sistema electoral enteramente proporcional, sin prima electoral, cerrando definitivamente la hipótesis de un modelo de Westminster; un hecho que habría sido de todos modos semi-manifestado por carecer en todo caso de un elemento esencial: la legitimación política mutua en la esfera gubernamental.

Por otro lado, cuestiones de política internacional, podemos apuntar por ejemplo la invasión soviética de Hungría en 1956, apuntalaron la idea de continuar con esa proporcionalidad y no apostar por un sistema mayoritario, no en vano el Partido Comunista italiano con este último sistema podría llegar al gobierno de la Nación. A mayor abundamiento, la decisión de un sistema totalmente proporcional también sirvió para ampliar la base democrática, gradualmente separando — cómodamente y sin riesgo para nadie- el Partido Socialista del Partido Comunista, marginando aún más a este último. Un hecho que tuvo la oportunidad de dar dinamismo a un sistema político establemente basado exactamente en la política de la Democracia cristiana, en el gobierno desde 1948 hasta 1993.

Sin embargo, precisamente porque las propias fuerzas políticas sabían bien que las razones de apostar por un sistema proporciona a nivel nacional estaban vinculadas a un contexto específico y, por lo tanto, eran potencialmente transitorias, la opción por un sistema electoral proporcional no se constitucionalizó ${ }^{10}$.

El sistema electoral proporcional, cristalizado definitivamente en 1957 con la aprobación del texto único sobre las leyes electorales (Decreto presidencial del 30 de marzo de 1957, n. 361), representó entonces la consideración de la inmutabilidad en el posicionamiento —interno e internacional- de Italia, mientras, por otro lado, fue la traducción del conventio ad excludendum del Partido Comunista del área de gobierno, que luego fue delineado en doctrina por Leopoldo Elia. ${ }^{11}$

Desde entonces nada aconteció hasta básicamente los años ochenta. Hubo diferentes propuestas de reforma constitucional, todas fallidas, y se crearon varias comisiones parlamentarias ad boc para valorar la reforma del sistema.

A partir de entonces se comenzó a cambiar hacia un mayoritarismo funcional (aún no estructural), con algunas innovaciones, como, por ejemplo, las reformas

9 La Ley n 148 de 31 de marzo de 1953 asignaba un premio mayoritario sólo a la Cámara de Diputados, a la lista o listas vinculadas entre sí que, en todo el territorio nacional, habían obtenido 50,01 de los votos. Las elecciones políticas generales del 7 de junio de 1953 se celebraron, por tanto, para el Senado con la Ley $\mathrm{n}^{\circ} 29$ del 6 de febrero de 1948, y para la Cámara con la nueva ley. Las fuerzas políticas de la coalición mayoritaria obtuvieron el 49.2 no aprovechando el premio de la mayoría y anulando los efectos de la ley que luego fue derogada con la Ley $\mathrm{N}^{\circ} 615$ de 31 de julio de 1954, que restauró en todas sus partes las normas del texto único de 1948.

10 Por otro lado, donde las divisiones sociales eran étnicas y lingüísticas, es decir, tendencialmente permanentes, la Asamblea Constituyente las consagró formalmente, no por casualidad, en los estatutos especiales. Sin embargo, la tesis de Carlo Lavagna es bien conocida — ahora definitivamente desactualizada-, que considera la elección del sistema electoral proporcional intrínsecamente vinculado con el sistema republicano, de hecho, potencialmente implícito en el sistema de la propia Carta Constitucional. Vid. C. Lavagna, Il sistema elettorale nella Costituzione italiana, Rivista Trimestrale di Diritto pubblico, 3, 1952, pp. 849 y ss.

11 Vid. L. ElIA, Governo (forme di), Enciclopedia del Diritto, vol. XIX., 1970, ad vocem. 
regulatorias que, desde 1988 en adelante, redujeron el voto secreto en Parlamento y le dieron al gobierno, junto con el Presidente de la Cámara, un papel significativo en la planificación del trabajo parlamentario (aunque muy débil, si se considera en una clave comparativa), o con la nueva ley del Gobierno, la Ley n. 400 del 23 de agosto de 1988 y la simplificación de los procedimientos presupuestarios.

Estas herramientas, que predijeron la crisis de proporcionalidad, ofrecieron al sistema de partidos la oportunidad de captar el cambio en curso. Pero esto no sucedió con la profundidad y amplitud de la visión de intervención que hubiera sido necesaria.

Por lo tanto, aunque en términos constitucionales, la necesidad percibida de cambio resultará en un ritmo más intenso en las revisiones del texto constitucional (más puntuales y específicas, pero inconsistentes o irrelevantes con respecto a una visión general), desde el punto de vista del sistema de partidos políticos se produce una pulverización progresiva de las fuerzas políticas que construyeron la República, dejando al sistema político-constitucional más y más débil, al borde de una verdadera crisis de realineación errado entre el sistema político-constitucional y el sistema electoral. Debilitado también por los embates de un movimiento político, la Liga del Norte, que luego rompió la forma de estado, a través de propuestas de secesión, el sistema político italiano de los llamados Primera República comenzó a derrumbarse.

La crisis de la lógica operativa proporcional, típica de una democracia consensual y consociativa, se produce en varios niveles: a través de la nueva ley sobre la organización del autogobierno local (ley 8 de junio de 1990, n. 142), que fortalece una relación más directa entre los ciudadanos y las administraciones locales; siguiendo el resultado del primer referéndum electoral del 9 de junio de 1991, que pidió la abrogación de la preferencia múltiple en la ley electoral de la Cámara de Diputados, produciendo así un mecanismo proporcional con una sola preferencia que habría sido utilizada solo en 1992; finalmente, con la explosión, desde febrero de 1992, de la investigación del poder judicial sobre los fenómenos de corrupción (los llamados «Tangentópoli»).

Estos tres eventos, cada uno a su manera, socavaron el sistema de partidos políticos que habían fundado la Italia republicana, basada en una lógica proporcional y consociativista.

También hubo otros dos factores que son necesarios apuntar.

Primero, la grave crisis económica de 1991-1992, vinculada al proceso de reunificación alemana y al fortalecimiento del proceso de integración europea (Maastricht), así como por causa de la elevada deuda pública que redujo drásticamente posibilidad de utilizar fondos públicos ilimitados ${ }^{12}$. Por lo tanto, reducida la posibilidad de depender de recursos financieros que, sin responsabilidad ni

12 Vid. G. Амато, Un governo nella transizione. La mia esperienza di Presidente del Consiglio, Quaderni costituzionali, 3, 1994, pp. 355 ss. 
límites, podrían ser distribuidos por el sistema de partidos políticos (también a través del mecanismo de voto de preferencia, que era el otro lado del sistema electoral «a la italiana»), la lógica proporcional que había dominado durante más de cuarenta años llegó a desgastarse rápidamente.

El segundo factor se produjo casi al mismo tiempo, al amparo del referéndum de abril de 1993, en orden a introducir un sistema sustancialmente mayoritario, Se derogaron, asimismo, artículos de la ley el 2 de mayo de 1974, n. 195, con respecto a la «Contribución del Estado a la financiación de los partidos políticos», con el objetivo de eliminar partidas de la financiación pública y directa para los partidos políticos.

\section{EL SISTEMA ELECTORAL ITALIANO EN LA SEGUNDA FASE REPUBLICANA (1994-2020): VEINTISÉIS AÑOS INTENSOS, ENTRE YA Y TODAVÍA NO}

La segunda fase republicana (1994-2020) se caracteriza por una profunda inestabilidad gubernamental, no en vano ha habido dieciséis gobiernos y se han celebrado siete elecciones generales; pero, sobre todo, lo hace también por el nacimiento de un segundo sistema de partidos políticos, completamente nuevo en comparación con el anterior.

Todo ello llevó a que la cuestión de la ley electoral fuera muy relevante en este período y que haya estada fuertemente entrelazada con la reforma de la estructura constitucional del país, con sus intentos de modificarla y con las oportunidades perdidas: retos fallidos que han contribuido en gran medida a la inestabilidad y al fracaso de la consolidación de un nuevo sistema de partidos y, con él, una nueva ley electoral que fuera duradera, estable y eficiente.

En este contexto, la lógica mayoritaria del sistema electoral introducida en el 1991-1993 en las «ramas altas» del orden — tomando prestada la conocida metáfora de Giuliano Amato del orden y de la estructura constitucional como un árbol - ya se había arraigado muy positivamente en las ramas inferiores, las de la autonomía política.

De hecho, tanto la ley de elección directa del alcalde (Ley $\mathrm{N}^{\circ} 81$ de 1993), como la ley para regular el sistema electoral de las regiones de estatuto ordinario (Ley $\mathrm{N}^{\circ} 43$ de la 1995), que garantizaba, a través de una lista de precios vinculada, una prima mayoritaria de al menos el $55 \%$ de los escaños a la coalición ganando (y previó, como garantía de gobernabilidad, elecciones anticipadas ante una crisis en los primeros dos años «regla anti-vuelco»), sacó a relucir una lógica de funcionamiento de tipo bipolar y mayoritario.

Esto provocó que las instituciones locales y sus representantes, tanto en lo que respecta a la función como a la funcionalidad político, actuaran más rápido respondiendo a los problemas que se venían sucediendo y, al mismo tiempo, 
estuvieran más cerca de las necesidades de la ciudadanía en comparación con lo que estaba sucediendo en su lugar en el sistema estatal.

Así, entre 1999 y 2001, mediante dos leyes de reforma constitucional que, continuaban por el camino ya abierto, se introdujo la elección directa de los presidentes de las regiones con estatuto ordinario y especial (l. cost. $\mathrm{n}^{\circ} 1$ de 1999 y $\mathrm{n}^{\mathrm{o}} 1$ de 2000), y se intentó estabilizar al menos la forma de gobierno local y regional ${ }^{13}$, favoreciendo también, gracias a la coherencia política de la intervención sobre las instituciones y el sistema electoral de las autonomías, el desarrollo de una mayor conciencia en el votante y una mayor responsabilidad de los administradores público; por lo tanto, un crecimiento real de la conciencia cívica y política ciudadana, que ha permitido que una nueva clase política emergiera.

La comparación entre los sistemas local y nacional se fue poniendo de manifiesto elección tras elección. La ciudadanía observó así que, aunque no parecía posible experimentar el verdadero sistema mayoritario a nivel nacional, el sistema regional italiano se estabilizaba, forma regional «de la transición» ${ }^{14}$, basado sobre una lógica mayoritaria, permitiendo gobernar las autonomías en un esquema típico de un parlamentarismo fuertemente racionalizado.

Consecuentemente, estos veintiséis años, nos muestran un panorama sin gobiernos estables a nivel nacional, consecuencia de un sistema de leyes electorales inconsistentes y dominado por una deslegitimación política recíproca entre las fuerzas políticas. Y, a la par, un nivel sub-nacional con alternancias naturales de gobierno sin crisis políticas.

La razón de esto bipolarismo nacional incoherente, tan diferente de lo de las autonomías sub-nacionales, es muy simple: para las autonomías, el cambio en la ley electoral también ha correspondido a un cambio en la forma de gobierno principalmente a través de la elección directa del alcalde o del Presidente de la Región y la reestructuración de la relación de confianza; en cambio, para el nivel nacional, a el cambio en la ley electoral nunca ha sido igualado por el cambio en la forma de gobierno a través de reformas constitucionales consistentes precisamente con la lógica de las leyes electorales adoptadas; de hecho, a pesar de numerosos intentos, incluidos dos referendos constitucionales fallidos (2006 y 2016), nunca ha sido posible implementar un cambio constitucional en la forma de gobierno nacional como para estar en línea con las nuevas opciones estratégicas que el nuevo sistema de las fiestas parecían tener la intención de posar, definiendo, vía, en pasar del tiempo, una nueva ley electoral.

La falta de correspondencia, por lo tanto, entre las reformas electorales y constitucionales ha debilitado el sistema de representación política, pero progresivamente ha hecho también más incierto en su dinámica de movimiento el

13 Vid. A. Chiaramonte - G. Tarli barbieri (Ed.), Riforme istituzionali e rappresentanza politica nelle Regioni italiane, Bologna, il Mulino, 2007

14 Vid. M. CARLi - C. Fusaro, Elezione diretta del Presidente della Giunta regionale e autonomia statutaria delle Regioni, Bologna, Il Foro Italiano-Zanichelli, 2002. 
sistema institucional, siempre comprimido entre la lógica «gobernante», típico de la modernidad de hoy y el azar de leyes electorales realizadas deliberadamente para ser incapaces de durar realmente.

En todas las legislaturas de los últimos veintidós años, ningún gobierno postelectoral ha sido capaz de durar toda la legislatura. De hecho, cada gobierno, después de aproximadamente un año, ha sido reemplazado por gobiernos formados en el Parlamento, no pocas veces consecuencia del transfuguismo parlamentario. La única excepción la encontramos en la XIV legislatura que, a pesar de haber visto varios gobiernos, todos ellos fueron dirigidos por el mismo Primer Ministro, S Berlusconi.

Naturalmente, tal situación no podía dejar de favorecer el surgimiento de partidos populistas, por un lado, y formas importantes de intervención de los diversos Presidentes de la República con el objetivo de «corregir» los efectos de la dinámica de una forma cada vez más débil de gobierno parlamentario ${ }^{15}$.

\section{La Ley Mattarella (1994-2005): un sistema electoral mixto}

Tras un primer referéndum sobre la reducción de las preferencias que pueden expresarse para la elección de diputados y la posibilidad de expresar la preferencia con indicación del número de listas, que se celebró con resultado positivo en junio de 1991, como se mencionó anteriormente, en abril de 1993 se celebró un segundo referéndum para derogar determinadas disposiciones de la ley electoral del Senado (Ley No 29 de 1948 y enmiendas posteriores) y eliminar la norma que preveía la elección en la circunscripción uninominal sólo después de obtener un elevado quórum del $65 \%$ de los votos, de lo contrario se determinó la distribución de los votos sobre una base proporcional.

Después del referéndum, en pleno colapso del sistema de partidos de la época se reformó rápidamente el sistema electoral, adoptando dos nuevas leyes electorales para el Parlamento con una implantación de mayoría clara: las leyes del 4 de agosto de 1993 n. 276 y n. 277, llamadas leyes Mattarella o «Mattarellum», del nombre del primer firmante del proyecto de ley el diputado Sergio Mattarella (actualmente Presidente de la República Italiana desde 2015).

Se articulaba así un sistema electoral mixto tanto para el Senado como para la Cámara ${ }^{16}$, que se caracterizaba por la elección de las tres cuartas partes de los diputados y las tres cuartas partes de los senadores con un sistema de mayoría de

15 V. Lippolis - G. M. Salerno, La presidenza più lunga. I poteri del capo dello Stato e la Costituzione, Bologna, Il Mulino, 2016. Y tambien, v. F. CLementi, Garante o governante? La figura del Capo dello Stato nella recente esperienza dei Paesi dell'Unione europea a regime repubblicano, en Diritto pubblico comparato ed europeo, $\mathrm{n}^{\circ} 3$, 2016, pp. 617-638.

16 Ley No 276 de 4 de agosto de 1993 (sobre el Senado) y la Ley No 277 de 4 de agosto de 1993 (sobre la Cámara). 
una sola ronda en circunscripciones únicas. Los escaños restantes se asignaron por el sistema proporcional: en la Cámara, distribuyéndolos, en las veintiséis circunscripciones electorales, entre las listas competidoras que habían superado la barrera del $4 \%$ de los votos en el ámbito nacional; en el Senado, distribuyéndolos entre grupos de candidatos en proporción a los votos obtenidos en las circunscripciones de cada región por los candidatos no elegidos. ${ }^{17}$

En cuanto a los métodos de votación, a diferencia del Senado, el elector emitió dos votos para la Cámara en dos votaciones diferentes: uno para los candidatos de las circunscripciones uninominales y otro para las listas que contribuyeron a la distribución de los escaños sobre una base proporcional.

Los candidatos se presentan en los colegios para la parte mayoritaria y en las circunscripciones para la parte proporcional, siendo, como se ha dicho, un sistema mixto. Sin embargo, se establecían vínculos entre los dos partidos, ya que los candidatos de las circunscripciones unipartidistas tenían que estar vinculados a las listas proporcionales de la circunscripción y podían, en casos particulares, ser elegidos por la parte proporcional. Excluidas del reparto quedarían las listas que no obtuvieran al menos el $4 \%$ de los votos válidos en todo el territorio nacional. Las listas, por su parte, participaron en la distribución sólo después el «scorporo»: un mecanismo que proporcionó que restara, del recuento de los votos totales de una lista en la parte proporcional, los votos obtenidos por los candidatos conectados a la misma lista elegidos en los colegios uninominales con el sistema mayoritario ${ }^{18}$.

Sin embargo, el bipolarismo que surge de las primeras elecciones políticas del 27 al 28 de marzo de 1994 con la vitoria de S. Berlusconi asume inmediatamente las características de una radicalización ideológica, alimentando un clima de conflicto político-ideológico permanente, a pesar de la caída del Muro de Berlín y fin, de hecho, de las llamadas ideologías de masas.

El mismo sistema electoral también contribuyó a este clima de confrontación porque, por un lado, presentaba el sistema de tipo mayoritario mencionado anteriormente, que comparaba coaliciones alternativas, y por otro estaba

17 En particular, con la Ley N²77, de 4 de agosto de 1993, las normas para la elección de la Cámara de Diputados se modificaron fuertemente y las nuevas reglas condujeron a la elección de 475 diputados por el sistema de la mayoría en otras tantas circunscripciones uninominales; en cambio, 155 fueron elegidos por el sistema proporcional, es decir, distribuyéndolos en proporción a los votos obtenidos de las listas competidoras presentadas en las 26 circunscripciones.

18 Posteriormente dos leyes de revisión constitucional (17 de enero de 2000, No 1 , y 23 de enero de $2001, N^{o} 1$ ) otorgaron a los ciudadanos italianos residentes en el extranjero el derecho a elegir, dentro de una circunscripción extranjera, seis senadores y doce diputados. Dado que el número total de miembros de las dos Cámaras se mantuvo sin cambios, el número de escaños a distribuir en las circunscripciones nacionales - menos los que se asignarán en la Circunscripción Extranjera- se redujo por lo tanto a 618 para la Cámara y 309 para el Senado. La Ley No 459, de 27 de diciembre de 2001, puso en práctica la disposición constitucional que regula el ejercicio del voto (por correspondencia) y la asignación (por sistema proporcional) de los escaños asignados a la circunscripción extranjera. Vid. mas recientemente, M. FeDI - G. GAmbardelLA, $I l$ voto degli italiani all'estero: dalle origini del dibattito alle problematiche attuali fino alla recente pronuncia (di rito) della Corte Costituzionale sulle modalità di voto, Rassegna dell'Avvocatura dello Stato, $\mathrm{n}^{\circ} 1,2018, \mathrm{n}^{\circ} 1, \mathrm{pp} .265$ ss. 
contaminado por la presencia de la cuota proporcional, que por el contrario exaltó el espíritu de identidad de los partidos, a expensas de las coaliciones.

La disposición combinada de votar por la coalición y votar por el partido, que fueron contextuales, ayudaron a multiplicar la sensación de distanciamiento y confusión del elector. Su desorientación se vio agravada por el hecho de que, en la fase que precedió a las elecciones, naturalmente se sometió a dos campañas electorales, contemporáneas y distintas: la mayoría, para la coalición, de tipo bipolarista y uninominal; y el proporcional, para la lista de partes, de un tipo de identidad de parte.

Por lo tanto, entre coaliciones construidas más por conveniencia que por convicción, en las tres elecciones celebradas (27-28 de marzo de 1994; 21 de abril de 1996; 13 de mayo de 2001) el sistema político ha sufrido fluctuaciones muy fuertes, con el único efecto de corroerse cada vez más gradualmente el sistema mayoritario y el apoyo social y político que había dado lugar a las leyes electorales de Mattarella.

Por supuesto, dentro de dentro de estos procesos transicionales, los efectos de las leyes electorales deben trasladarse también sobre los Reglamentos parlamentarios. Así, de 1997 a 1999, conscientes del impulso, los partidos intentaron varias veces reformar las asambleas legislativas para reconstruir sobre el eje de la bipolarización un nuevo Parlamento ${ }^{19}$, llegando incluso a imaginar la institucionalización del papel de la oposición y su líder, primero como líder de coalición ${ }^{20}$ y luego como líder en sentido estricto ${ }^{21}$. Y, sin embargo, las reglas no podrían haber obligado más que el propio comportamiento político.

Sobre la base de estas reglas electorales, divididas entre las identidades de los partidos y la lógica coalicional, los partidos nunca lograron reducir la fragmentación de los partidos, sobre todo por su tendencia a dividirse a lo largo de líneas de fractura intra-coalicional en lugar de coalicional. Esta tendencia fue alimentada, como hemos dicho, por los mecanismos e instrumentos propios de las leyes de Mattarella, ya que incluso la propia duplicación de la elección en dos votos —en las dos papeletas separadas que recibió cada elector, una para el voto en el colegio mayoritario y otra para el proporcional — garantizaba el mantenimiento de las identificaciones tradicionales de los partidos en una lógica esquizofrénica y

19 Vid. L. Violante (Ed.), Il Parlamento, Storia d'Italia, XVII, Torino, Einaudi, 2001; S. CeccantI, Regolamenti parlamentari: un altro tassello di una riforma «strisciante», Quaderni costituzionali, 1998, $\mathrm{n}^{\circ} 1$, pp. 157 ss.; F. Clementi - E. Gianfrancesco, L'adeguamento dei regolamenti parlamentari al sistema bipolare, en Per far funzionare il Parlamento. Quarantaquattro modeste proposte, por F. BASSANINI - A. MANZELLA, Quaderni di Astrid, Bologna, il Mulino, 2007, pp. 35 ss.; A. MANZElla (Ed.), I regolamenti parlamentari a quarant'anni dal 1971, Bologna, il Mulino, 2012, F. LANCHESTER (Ed.), Regolamenti parlamentari e forma di governo: gli ultimi quarant'anni, Quaderni di Nomos, Milano, Giuffrè, 2013.

20 Vid. E. GuARDuCCI, La rappresentanza unitaria di coalizione, in Rappresentanza politica, gruppi parlamentari, partiti. II. Il contesto italiano, por S. MERLINI, Torino, GiappiCHELli, 2004, pp. 213 ss

21 G. Perniciaro, L'istituzionalizzazione del ruolo dell'opposizione: profili organizzativi. In La riforma dei regolamenti parlamentari al banco di prova della XVI Legislatura, por E. GIANFrANCESCO - N. LupO, Roma, Luiss University Press, 2009, pp. 95 ss. 
contradictoria, que no podía dejar de generar desorientación, así como asimetrías de comportamiento, tanto del lado de los partidos como del de los electores.

\section{La Ley Calderoli (2005-2016): un proporcional con premio mayoritario}

Al final de la XIV legislatura (30 de mayo de 2001 - 27 de abril 2006), con el único apoyo de la mayoría de centro-derecha, y bajo el fuerte impulso del Ministro de la Reforma, el senador Roberto Calderoli, se aprueban, por un lado, una reforma integral de la Segunda Parte de la Constitución y, por otro lado, como complemento de la reforma constitucional, una nueva ley electoral, la Ley $\mathrm{N}^{0} 270$ de 21 de diciembre de 2005, el llamado «Porcellum», como se llama periodísticamente a la ley Calderoli, que duró hasta el 2016.

Era un sistema electoral completamente proporcional, con la posible atribución de un premio mayoritario a nivel nacional. Disponía que los 617 diputados fueran elegidos en el territorio nacional en proporción a los votos obtenidos por las listas en competencia presentadas en los 26 distritos electorales (con la excepción de 1 elegido con método mayoritario en el colegio uninominal del Valle de Aosta y los 12 diputados restantes elegidos en el distrito extranjero), mediante los procedimientos establecidos por la ley n 459 de 27 de diciembre de 2001, y por el reglamento relativo de implementación (Decreto presidencial no 104 de 2003).

Las nuevas regulaciones establecieron que los partidos políticos que deseasen presentar listas de candidatos podrían unirse en coaliciones; mientras que los partidos que se postularon para gobernar también deberían haber depositado su programa e indicar el nombre de su líder. Se brindó la posibilidad de que el elector emitiera solo un voto para la lista elegida, sin ningún voto de preferencia; y los escaños se distribuyeron proporcionalmente a nivel nacional entre las coaliciones de listas y las listas que habían excedido las cláusulas de barrera.

El punto más importante era que la coalición de listas más votada (o la lista no coaligada), si no había obtenido ya al menos 340 escaños, recibía un premio mayoritario de tal manera que se garantizaba la mayoría absoluta de escaños.

Este sistema electoral, que ha regido tres elecciones generales (9-10 de abril de 2006; 13-14 de abril de 2008; 24-25 de febrero de 2013), fue la símbolo del retorno del «poder del partido» ${ }^{22}$, marcando en primer lugar la transición de un sistema casi enteramente mayoritario a uno de tipo proporcional, con un evidente y progresivo debilitamiento de los elementos típicos de una competición realmente bipolarista y de alternancia.

Sin embargo, el funcionamiento de este nuevo sistema electoral se reveló inmediatamente problemático, precisamente por la adjudicación del premio de

22 Vid. F. LANCHESTER, I diritti di partecipazione politica nell'innovazione istituzionale incrementale italiana, Federalismi.it: rivista di diritto pubblico italiano, comunitario e comparato, 2006, p. 23. 
mayoría: de hecho, si la atribución del premio era segura para la Cámara de los Diputados, la misma atribución era incierta para el Senado, siendo el premio otorgado sobre «una base regional» (art. 57 Const.) y, por lo tanto, no previsible a priori. La atribución del premio se convirtió en una suerte de lotería ${ }^{23}$, así como la posibilidad de tener una mayoría del mismos signo político en ambas Cámaras. Por lo tanto, desde las elecciones de 2006, la dificultad de atribuir, región por región, los premios individuales previstos en la fórmula electoral para el Senado demostraron una vez más el papel clave de los partidos menores como factor de inestabilidad para el gobierno.

Por otro lado, como se mostró sobre todo en las elecciones de 2008, este también era un sistema electoral esquizofrénico: antes de las elecciones, estimuló la agregación de fuerzas políticas de la coalición, con el objetivo de obtener la mayor cantidad posible de premios regionales para el Senado; luego, después de las elecciones, favoreció la fragmentación de las mismas coaliciones en el Parlamento, ya que una vez que los partidos menores ingresaron en el Parlamento gracias a la superación de los bajos umbrales previstos para los partidos que formaran parte de una coalición, pudieron retener a los partidos principales como rehenes, utilizando todo su blackmail power (poder de chantaje) para condicionar la línea política del gobierno.

En resumen, nuevamente, se desplegaron mecanismos de fragmentación de los partidos políticos con el objetivo de debilitar el bipolarismo, haciendo que sea aún más difícil la celebración de los rendimientos de este sistema.

También hubo otros defectos: el de un premio mayoritario que, sin límite, podría ser excesivamente desproporcionado en circunstancias políticas concretas, como luego señalaría la Corte constitucional en la sentencia $n^{\circ} 1$ de 2014 . A esto se sumó el defecto de romper el vínculo entre electores y elegidos mediante el establecimiento de listas de partidos cerradas y bloqueadas, confiándose solo a los líderes del partido las elecciones de los candidatos, así como su elegibilidad (reduciendo en gran medida la calidad de los elegidos, que en su mayoría se convierten en leales o complacientes con el líder), a su vez, otro punto que después fue criticado por la Corte con la sentencia $n^{\circ} 1$ de 2014 . Y luego, el de un procedimiento electoral desigual, porque permitió que aquellos que ya estaban representados pudieran renunciar a la recolección de firmas para la presentación de candidatos.

Por lo tanto, a pesar del cambio de estrategia política de los partidos con el nacimiento de nuevas formaciones políticas «con vocación mayoritaria» (ya que en 2007 nació el «Partido Democrático», a la izquierda y, a la derecha, el

23 Vid. A. Gratteri, Genesi di un precedente: l'interpretazione dell'elezione a base regionale del senato da parte del Presidente Ciampi, Nomos. Le attualità nel diritto, $\mathrm{n}^{\circ}$ 3, 2017. Para un comentario completo y actualizado sobre el problema técnico-político del artículo 57 de la Constitución, véase E. Gianfrancesco, Commento all'articolo 57, en F. Clementi, L. Cuocolo, F. Rosa, G.E. Vigevani (Eds.), La Costituzione italiana. Commento articolo per articolo, Bologna, Il Mulino, 2018, vol. I-II. 
«Pueblo de las Libertades ${ }^{24}$ ), la ley Calderoli fue incapaz de evitar algunos problemas tradicionales, como el aumento de la fragmentación política, la permanencia de las coaliciones formadas para interés y no por convicción. Además, supuso un aumento dramático de la distancia entre los elegidos y los votantes, dispersando el valor del vínculo con el territorio, que al menos en la ley de Mattarella había mejora por la división en colegios uninominales, y, tras la experiencia de tres elecciones, fue criticado por los votantes.

Sin embargo, el nacimiento del Partido Demócrata y el Pueblo de la Libertad no impidió que el sistema político siguiese empeñado en trabajar, en un proceso de vuelta al pasado, en torno a propuestas de leyes electorales, casi todas ellas proporcionales, declinadas, a lo sumo, hacia aquellos modelos proporcionales que todavía «tienen un efecto mayoritario», como, por ejemplo, el sistema electoral español ${ }^{25}$.

Así que, dentro de una dinámica de gobierno en perpetua agitación y sobre la base del resultado negativo de las elecciones europeas y locales de 2009 y de las elecciones regionales de 2010, a lo largo de un conflicto cada vez más evidente entre los dos dirigentes del partido único «Pueblo de la Libertad», Berlusconi y Fini, y de las dificultades de Walter Veltroni, líder del Partido Demócrata, para superar la resistencia interna de su partido a fin de caracterizarse cada vez más como un partido de oposición al modelo Westminster, se llega rápidamente a la división del recién nacido Pueblo de la Libertad, entre Fini, que dará vida al movimiento «Futuro y Libertad», y Berlusconi, acusado por la Fiscalía de Milán de extorsión y prostitución infantil, que después sufrirá también una derrota en la las elecciones locales de 2011.

La rápida crisis del gobierno de Berlusconi conduce al nacimiento del gobierno de Monti en noviembre de 2011, un momento sin retorno: tanto porque a partir de ese momento no habrá, a pesar de las diferencias individuales, gobiernos derivados de la «elección directa», y porque esta situación muestra un estancamiento político-institucional muy evidente.

Es cada vez más claro, por lo tanto, que casi veintiséis años después del cambio en el régimen político, entre el bipolarismo y la fragmentación, este último sigue siendo el elemento más importante para las fuerzas políticas, que no tienen la intención de renunciar a la posibilidad de formar gobiernos en el Parlamento, en lugar de tomar nota de los resultados electorales que, también en términos de gobernabilidad, determinaron los electores con su voto.

24 C. Fusaro, Dalle coalizioni-cartello ai partiti a vocazione maggioritaria: un passo verso la governabilità?, in Quaderni costituzionali, 2, 2008, pp. 359 y ss.

25 Vid. T.E. Frosini, Una legge elettorale che salvi il bipolarismo, en Percorsi costituzionali, 2011, n ${ }^{\circ} 1,1$ ss.; S. FABBRINI, Salvare il bipolarismo e ridurre la frammentazione, en Mulino, 2008, $\mathrm{n}^{\circ}$ 1, p. 66; más ampliamente, A. Chiaramonte, Il nuovo sistema partitico italiano tra bipolarismo e frammentazione, en R. D'Alimonte, A. Chiaramonte (Ed.), Proporzionale se vi pare. Le elezioni politiche del 2008, Bologna, il Mulino, 2010, pp. 392 y ss. 


\section{UNA TERCERA FASE? GOBIERNOS POPULISTAS Y SISTEMAS ELECTORALES. (2016-2018)}

El cambio y la estabilización del régimen político se intentaron, una vez más, como en 1991-1993, desde fuera del sistema de partidos, eligiendo nuevamente el camino del referéndum para modificar, en parte o en su totalidad, la ley $\mathrm{n}^{\circ} 270$ del 21 de diciembre 2005, para cerrar definitivamente la transición político-institucional abierta entre 1994 en un sentido mayoritario y bipolarista.

Un primer intento fue el referéndum del 21 y 22 de junio de 2009, establecido para contener la fragmentación y evitar coaliciones «monstruo»: tenía como objetivo limitar el premio mayoritario a las listas solamente, es decir, sin extenderlo también a las coaliciones, y quería abolir las candidaturas múltiples, la verdadera causa de lo que comenzaba a llamarse el «Parlamento de los nominados». Sin embargo, este referéndum falló debido a la falta de quórum (solo participó el $23.52 \%$ de los votantes).

Posteriormente, se promovió otro referéndum con el objetivo de derogar toda la ley Calderoli, queriendo determinar — como efecto del «sí»— la reactivación de las leyes de Mattarella de 1993, siguiendo la doctrina jurídica de la «reviviscencia de las normas». En este caso, sin embargo, fue la propia Corte Costituzionale, el 12 enero de 2012, la que declaró inadmisible el referéndum porque, a su juicio, lejos de producir el resurgimiento del texto que estaba antes de la ley de Calderoli, habría dejado un vacío regulatorio que su jurisprudencia consideraba un motivo de inadmisibilidad (siempre que se refiriese a órganos constitucionalmente necesarios).

Entonces, después de la renuncia del IV gobierno de Berlusconi, el nombramiento del profesor Mario Monti como senador vitalicio y su posterior designación para formar un nuevo gobierno. Un gobierno que se formó precisamente para evitar elecciones anticipadas que el presidente de la República, Giorgio Napolitano, consideró perjudiciales para los intereses del país en un momento en que, después del estallido de la crisis financiera, el coste de la enorme deuda pública acumulada corría el riesgo de multiplicarse hasta que volverse insostenible. Precisamente ese gobierno, el gobierno de Monti, abrió una nueva etapa de transición, la de un gobierno de técnicos, apoyado por una mayoría compuesta por el centro-derecha y por la ya oposición de centro-izquierda contra el gobierno Berlusconi.

Un gobierno que tanto salvó a Italia y sus finanzas públicas como ha sido «viento en las velas» del populismo, y en particular del principal partido populista italiano, que acababa de nacer: el Movimento Cinque Stelle. En cualquier caso, el nacimiento del gobierno de Monti, reafirmado reiteradamente también en público por el propio presidente Napolitano, quería que las fuerzas políticas en el Parlamento aprobasen todas esas reformas necesarias, tanto institucionales 
como electorales, para dar a Italia representación política y estabilidad del gobierno, mientras que el ejecutivo se enfrentaba a la emergencia económica ${ }^{26}$.

Sobre esta base era evidente para todos que la ley electoral de 2005, entonces, se mostraba progresivamente cada vez más inadecuada, debido, en primer lugar, a la incongruencia de prever dos mecanismos de premio de mayoría distintos relacionados con dos Cámaras diferentes, votadas por diferentes electores, ambas titulares de la relación fiduciaria con el gobierno. De hecho, la combinación de la ley de Calderoli con el doble voto de confianza necesario para la formación del gobierno, previsto en la Constitución de 1948, hizo que la formación de una mayoría políticamente homogénea en ambas Cámaras fuera cada vez más difícil. Por otra parte, un sistema electoral de premios puede funcionar razonablemente para contribuir a la formación de un gobierno si se aplica a la composición de una sola asamblea. Pero es poco probable que ayude a un bicameralismo con una doble relación de confianza.

Sin embargo, no pasó nada. Y el estancamiento sistémico se evidenció claramente en las elecciones del $2013^{27}$, a pesar de la reelección del Presidente de la República Giorgio Napolitano -un unicum en la historia constitucional italiana-, que se había producido precisamente para favorecer una clara estrategia de reforma constitucional y electoral de las fuerzas políticas en el Parlamento. ${ }^{28}$

El sistema fue desbloqueado cuando la Corte costituzionale, con su sentencia $\mathrm{n}^{\mathrm{o}} 1$ de 2014, declaró la ilegitimidad constitucional de una parte de las disposiciones de la ley $\mathrm{n}^{\circ} 270$ de 2005 . Esto ha obligado a que el Parlamento apruebe un nuevo sistema electoral para la Cámara de Diputados, la ley no 52 de 2015, llamada Italicum, con un sistema proporcional con un premio mayoritario y una eventual segunda vuelta electoral.

Es importante decir que en la nueva ley electoral —el Italicum — no incidió sobre el sistema electoral del Senado, porque se confiaba en que se aprobaría la reforma constitucional — que fue rechazada en el referéndum del 4 de diciembre de 2016 - que preveía la transformación del Senado en un órgano electivo de segundo grado. Entonces, como todo intento de revisión constitucional y electoral del centro-derecha entre 2005 y 2006 fue destruido, también el intento de

26 No es casualidad que el gobierno de Monti fuera uno de los cuatro, en los últimos treinta años, sin un ministro responsable de la reforma constitucional y electoral. Vid. C. FusAro, La formazione del governo Monti e il ruolo del presidente della Repubblica, en Politica in Italia. Edizione 2012, por A. BosCO - D. MCDONNELL, Bologna, il Mulino, 2012, pp. 83 ss.

27 Los resultados de las elecciones demostraron que ninguna de las coaliciones fue capaz de lograr una victoria neta, lo que condujo a un resultado sin precedentes en la historia de las elecciones políticas italianas. Y el Movimento Cinque Stelle, fundado y dirigido por el comediante Beppe Grillo en 2005, que había logrado estar presente en 2012 en cien de las aproximadamente mil elecciones municipales, eligiendo cuatro alcaldes y, en particular, el de Parma, en la fragmentación progresiva del panorama político, en las elecciones políticas de 2013 obtuvo los $25.56 \%$ en la Cámara y $23.78 \%$ en el Senado, es decir, 109 diputados y 54 senadores.

28 F. Clementi, L'elezione del Presidente della Repubblica e l'eredità della Presidenza Napolitano, en Politica in Italia: i fatti dell'anno e le interpretazioni, C. Fusaro - A. Kreppel (Ed.), Bologna, Il Mulino, 2014. 
centro-izquierda, entre el 2013-2016, muy deseado por el Presidente del Consejo Matteo Renzi, tras el resultado del referéndum constitucional del 4 de diciembre de $2016^{29}$.

En este sentido, entonces, el populismo ha tenido un camino claro y muy fácil de crecimiento político, frente a la incapacidad del sistema de partidos tradicional para dar una respuesta adecuada en términos de reformas. De hecho, el fracaso de las reformas constitucionales y electorales con referencia particular a lo que concierne al funcionamiento del sistema político institucional, es decir, la forma de gobierno entendida estrictamente como un sistema de decisiones colectivas, por lo tanto, he dejado al sistema político cada vez más a merced del populismo y en particular del partido populista por excelencia, el Movimento Cinque Stelle; que, después de estar en la oposición durante toda la legislatura 20132018, en las elecciones de 2018 se convirtió en el primer partido político italiano, con más del $30 \%$ de los votos. ${ }^{30}$

Entonces, en coherencia con el resultado negativo del referéndum de diciembre de 2016, la Corte costituzionale, muy rápidamente, en su sentencia n 35 del 25 de enero de 2017, en particular, declaraba la ilegitimidad constitucional de las disposiciones de la ley 52/2015 (l'Italicum) que preveía una segunda vuelta electoral y las reglas que permitían a los candidatos elegidos en más de un colegio decidir a su discreción por el cual de ellos resultaban finalmente electos. La Corte dejaba así un sistema electoral puro.

En un completo reajuste, también para superar los dos sistemas electorales de la Cámara y el Senado, ambos con un sistema proporcional como resultado de la sentencia $\mathrm{n}^{\circ} 1$ de la Corte costituzionale de enero de 2014, que declaró ilegítimas partes del llamado Porcellum, válido para el Senado; y después de la sentencia $\mathrm{n}^{\circ} 35$ de la Corte costituzionale de enero de 2017, que declaró ilegítimas partes de la llamada ley electoral Italicum, válido para la Cámara de Diputados, en 2017 el Parlamento aprobó finalmente una nueva ley electoral de la Cámara de Diputados y el Senado de la República, la ley 3 de noviembre de 2017, n. 165, llamada Rosatellum bis o simplemente Rosatellum (por el nombre de su relator, el diputado Ettore Rosato), que luego se utilizó por primera vez en las elecciones del 4 de marzo de 2018.

En particular, esta ley — que está actualmente en vigor- describe un sistema electoral mixto, mayoritario y proporcional.

Precisamente, para la Cámara de los Diputados, el territorio nacional está dividido en 28 circunscripciones (en algunos distritos el territorio coincide con el de toda la región, mientras que en otros el territorio regional está dividido en varios distritos) y cada distrito está dividido en circunscripciones de un solo miembro y

29 Sobre el tema de las reformas constitucionales en Italia, ver resumen: C. FusAro, Per una storia delle riforme istituzionali (1948- 2015), en Rivista Trimestrale di Diritto Pubblico, 2, 2015, pp. 431.

30 En las elecciones políticas de 2018, el Movimento Cinque Stelle obtuvo el 32,68\% en la Cámara y el 32,22\% en el Senado, es decir, 225 diputados y 111 senadores. 
una o más circunscripciones de varios miembros ${ }^{31}$. Para la asignación del número restante de escaños, a prorrata, cada circunscripción se divide en circunscripciones de designación múltiple que consisten en la agregación del territorio de circunscripciones contiguas de designación única, de manera que a cada una de ellas se le asignen normalmente no menos de tres y no más de ocho escaños.

Con respecto a la elección del Senado, el territorio nacional se divide en 20 circunscripciones, correspondientes al territorio de cada región, que se dividirán también en circunscripciones uninominales y en una o más circunscripciones plurinominales. $^{32}$

Y para ambas Cámaras, el 37\% de los escaños (232 en la Cámara y 116 en el Senado) se asignan en un sistema de mayoría de una sola ronda en otros tantos colegios uninominales: en cada colegio se elige al candidato más votado, según el sistema conocido como uninominal (first past the post); el $61 \%$ de los escaños (386 y 193 , respectivamente) se asignan proporcionalmente entre las coaliciones y las listas individuales que han superado las cláusulas de barreras electoral nacional; la asignación de escaños se realiza a nivel nacional para la Cámara de los Diputados y a nivel regional para el Senado; para ello se crean colegios plurinominales en los que las listas adoptan la forma de listas bloqueadas de candidatos; en fin, el $2 \%$ de los escaños (12 diputados y 6 senadores) se destinan al voto de los italianos que viven en el extranjero y se asignan mediante un sistema proporcional que prevé el voto de preferencia.

Al mismo tiempo, la ley electoral dispone que cada lista debe presentar su propio programa y declarar su propio líder político, así como, si es necesario, la comparecencia con una o más listas para crear coaliciones: la existencia de una coalición, que es única a nivel nacional, obliga a las listas de la coalición a presentar un solo candidato en cada uno de los colegios. Existen diferentes cláusulas de barreras electorales: para listas no coaligadas, $3 \%$ de los votos obtenidos a nivel nacional; para listas únicas, $20 \%$ de los votos obtenidos a nivel regional (válido, alternativamente y sólo en el Senado); válido, alternativamente, para listas que representen a minorías lingüísticas reconocidas presentadas exclusivamente en regiones con estatuto especial en las que se ofrece protección especial a dichas minorías, el $20 \%$ de los votos obtenidos a nivel regional, o elección de dos candidatos en circunscripciones uninominales; para las coaliciones, siempre que incluyan por lo menos una lista que haya superado una de las otras cláusulas de barreras, $10 \%$ de los votos obtenidos a nivel nacional.

31 Los 231 colegios unipersonales del territorio nacional se dividen en cada distrito en función de la población (más el colegio unipersonal del Valle de Aosta).

32 Las 109 circunscripciones uninominales del territorio nacional se dividen en cada circunscripción en función de la población (más 1 circunscripción en el Valle de Aosta y 6 circunscripciones en Trentino-Alto Adige); y las circunscripciones de designación múltiple se componen de la agregación del territorio de circunscripciones contiguas de designación única, de manera que a cada una de ellas se le asignan normalmente no menos de dos y no más de ocho escaños. 
Para la determinación de la cifra electoral de la coalición (es decir, si se alcanza el 10\%), no contarán los votos emitidos a favor de las listas asociadas que no hayan obtenido al menos el $1 \%$ de los votos a nivel nacional; o, sólo en el caso del Senado, el 20\% a nivel regional; o, sólo en el caso de listas que representen a minorías lingüísticas reconocidas presentadas exclusivamente en regiones de estatuto especial en las que se prevea una protección especial de estas minorías, el 20\% a nivel regional o la elección de dos candidatos en circunscripciones uninominales.

Las listas vinculadas en una coalición que no alcancen la cláusula de barrera del $10 \%$ serán en todo caso admitidas a la distribución de escaños si han superado, según el caso, al menos una de las otras cláusulas de barreras.

Con respecto a la presentación de listas y nominaciones, cada partido o grupo político organizado que se proponga presentarse a las elecciones debe depositar su marca e indicar su nombre en el Ministerio del Interior en el plazo establecido. Al mismo tiempo que se deposita el símbolo electoral, se debe depositar también el programa electoral, en el que se declara el nombre y apellido de la persona indicada como jefe de la fuerza política; el estatuto del partido o, en su defecto, una declaración que indique ciertos elementos mínimos de transparencia. Naturalmente, los partidos o grupos políticos organizados pueden presentarse como una lista única o en coalición a nivel nacional presentando candidatos unificados en circunscripciones únicas. En cada colegio plurinominal, cada lista consiste en una lista de candidatos, presentados en un cierto orden numérico ${ }^{33}$.

Este sistema electoral considera el tema de las candidaturas múltiples y representación de género, y establece que ningún candidato podrá ser incluido en listas con la misma marca en más de 5 colegios plurinominales, a pena de nulidad de la elección. Todavía, el candidato en un distrito uninominal puede ser también un candidato en colegios plurinominales, con un límite de 5. Por último, el candidato del distrito electoral extranjero no puede ser candidato a la Cámara o al Senado. El diputado elegido en más de un colegio plurinominales será proclamado en el colegio en el que la lista a la que pertenezca haya obtenido el menor porcentaje de votos válidos en relación con el total de votos válidos del colegio. El miembro elegido en un colegio uninominal y en uno o más colegios plurinominales se considerará elegido en el colegio uninominal.

33 El número de candidatos de la lista no puede ser inferior a la mitad, redondeado al número entero más próximo, de los puestos asignados al colegio plurinominal y no puede exceder del número máximo de puestos asignados al colegio plurinominal; en todo caso, el número de candidatos no puede ser inferior a dos ni superior a cuatro. 


\section{V. ¿HACIA UN NUEVO SISTEMA ELECTORAL? LAS PROPUESTAS MÁS RECIENTES (2019-2020)}

Después de dos sentencias de la Corte costituzionale (sentencia no. 1 de 2014 y sentencia no. 35 de 2017); las elecciones de 2018 tampoco producen una mayoría en el Parlamento, con tres partidos ${ }^{34}$.

Una vez más, es gracias a la paciente mayéutica del Presidente de la República por lo que las fuerzas políticas logran formar un gobierno. Estos son inevitablemente gobiernos parlamentarios que viven, sin embargo, con un fuerte «correctivo presidencial», según la doctrina de Philippe Lauvaux ${ }^{35}$, dado que el Presidente de la República casi ejerce una función de «Lord Protector» del Presidente del Consejo.

Por lo tanto, ante la voluntad — que no parece superable por los partidos políticos- de que sean ellos los que formen los gobiernos en el parlamento después de la votación y, en cambio, no tomen nota del voto de los electores respetando su dirección política expresada en ese acto, en este punto los partidos parecen querer aprobar una ley plenamente proporcional para estabilizar el sistema político-institucional, tratando de volver a la llamada Primera República.

Sin embargo, el cambio del contexto político, social y económico hace que esta idea sea muy arriesgada, entre otras cosas debido al largo período de negociaciones entre las fuerzas políticas para formar alianzas; un largo período que las democracias modernas ya parece que no pueden permitirse.

En este contexto, como resultado de la aprobación de la reforma constitucional de la reducción del número de parlamentarios, que se votará en un referéndum constitucional popular el 29 de marzo de $2020^{36}$, se han puesto en marcha nuevas iniciativas legislativas de rango constitucional ${ }^{37}$ y ordinario, y también una reforma de la ley electoral para adaptarse obligatoriamente a las nuevas cifras previstas para el Parlamento (400 diputados en lugar de 630; 200 senadores en lugar de 315).

34 Los resultados han visto al centro-derecha establecerse como la coalición más votada, con alrededor del $37 \%$ de las preferencias, mientras que la lista más votada, el Movimento Cinque Stelle, obtuvo más del $32 \%$ de los votos. La participación en Italia fue del 72,93\% en la Cámara de Diputados y del 72,99\% en el Senado, lo que supone un descenso del 2,3\% respecto a las elecciones de 2013, siendo la más baja de la historia de la República Italiana (desde 1948).

35 Vid. P. Lauvaux - A. LE Divellec, Les grandes démocraties contemporaines, Paris, Presses Universitaires de France - P.U.F., 2015.

36 V. F. Clementi, Sulla proposta costituzionale di riduzione del numero dei parlamentari: non sempre «less is more», en Osservatorio sulle Fonti, n ${ }^{\circ} 2,2019$

37 En particular, se modifica el primer párrafo del artículo 57 de la Constitución, estableciendo que, con excepción de los escaños asignados a la circunscripción de Extranjero, el Senado será elegido «por circunscripción» y ya no, en cambio, por región, permitiendo así la posibilidad de que los senadores sean elegidos por varias regiones. Este es un factor decisivo, dadas las dificultades de representar territorios enteros, ya que ya no se dispone de hasta 115 senadores. Obviamente, se trata de reformas constitucionales obligadas por la entrada en vigor de la ley constitucional para reducir el número de parlamentarios. 
En particular, esta operación se llevó a cabo en primer lugar mediante la adaptación de la legislación vigente a un número reducido de parlamentarios gracias a la aprobación de la Ley $\mathrm{N}^{\circ} 51$ de 27 de mayo de 2019: una decisión que no fue «neutra» en términos de representación ya que determinó el número de escaños a asignar a partir de una relación fraccionaria, construida sobre los números actuales establecidos en la Constitución, alterando en gran medida la representación ${ }^{38}$.

Y luego, después de la inelegibilidad por la Corte costituzionale de la solicitud de referéndum popular presentada por el partido político de la Lega di Matteo Salvini, para cancelar completamente el sistema electoral en vigor y revivir la llamada Mattarella ${ }^{39}$, las fuerzas políticas del Parlamento, a partir de la segunda mitad de enero de 2020, han creado una iniciativa parlamentaria presentada por el Presidente de la Comisión de Asuntos Constitucionales, Giuseppe Brescia, que prevé 391 asientos asignados sobre una base proporcional, una barrera del 5\% y un «derecho de tribuna» para los que estén por debajo de la cláusula de barrera del $5 \%$.

Todavía parece ser — tanto en el fondo como en el método— sólo el comienzo de un proceso que, en mi opinión, no puede concluirse en poco tiempo, más allá de las palabras de los protagonistas.

\section{CONCLUSIÓN. EL SISTEMA ELECTORAL ITALIANO Y EL DESAFÍO DE LA CONSOLIDACIÓN.}

La ley electoral italiana ha sido en el último cuarto de siglo no sólo el método de transformación de votos en escaños sino el instrumento a través del cual los partidos políticos han intentado definir sus relaciones recíprocas de poder; ello, a pesar de que los propios partidos no tenían claro en sus estrategias en qué

38 Baste decir que las regiones y zonas más pobladas tendrán inevitablemente una ventaja sobre las menos pobladas, especialmente las que se encuentran dentro de ellas, mientras que no hay una representación igualitaria de todos los grupos lingüísticos en las regiones del Estatuto Especial y en las regiones que tienen minorías lingüísticas y culturales constitucionalmente garantizadas dentro de ellas.

39 Véase, en particular, la sentencia $\mathrm{N}^{\circ} 10$ por la que la Corte Constitucional declaró inadmisible la solicitud de referéndum popular sobre la «Abolición del método proporcional en la asignación de escaños en circunscripciones pluralistas en el sistema electoral de la Cámara de Diputados y el Senado de la República», presentada por los Consejos Regionales de Véneto, Piamonte, Lombardía, Friul-Venecia Julia, Cerdeña, Abruzos, Basilicata y Liguria.

40 V. Véase el proyecto de ley A.C. 2329, presentado el 9 de enero de 2020, cuyo examen se inició cinco días más tarde en la Comisión parlamentaria llevando por título «Modificaciones del texto único contemplado en el Decreto Presidencial No 361, de 30 de marzo de 1957, y del texto único contemplado en el Decreto Legislativo $\mathrm{N}^{\circ}$ 533, de 20 de diciembre de 1993, sobre la supresión de los colegios unipersonales y los umbrales de acceso a la representación en el sistema de elección de la Cámara de Diputados y el Senado de la República». Delegación en el Gobierno para la determinación de los distritos electorales multi-nominales». 
configuración institucional deseaban operar. En este sentido, el hecho de no desarrollar un cambio de la forma de gobierno a través de reformas constitucionales adecuadas ha provocado que todas las leyes electorales que se han adoptado hayan sido expuestas a presiones y vetos, que las han convertido en obsoletas muy rápidamente provocando su reforma al no corresponderse con las necesidades del momento. Fue un error constante considerar que una mera reforma electoral permitiría superar las dificultades y las ineficiencias político-institucionales del país, adoptando sistemas inevitablemente incoherentes o ineficaces. El reto de consolidar el sistema electoral en Italia sólo puede afrontarse si va acompañado simultáneamente de una reforma constitucional coherente de la forma de gobierno.

$$
* * *
$$

TitLE: The Italian Electoral System And Its Reform: The Challenge Of Consolidation

ABSTRACT: In the twenty-six years that characterize the last six Italian legislatures (1994-2020) Italy has seen, from the political-institutional point of view, an intense activity that was accompanied by a very similar vitality of the political party system. In this context, the electoral system and its continuous modifications with new electoral laws has strongly influenced the form of government, marking, by the decisive conditioning factor that represents the party system, its dynamics, both directly and indirectly. However, the rapid change of the different electoral systems in recent years has not been accompanied by an equal change in the constitutional text, so there has been an asymmetry in the functioning of the new electoral systems that were being adopted gradually, making them substantially incomplete, inconsistent and ultimately fragile. Faced with an immutability of the constitutional system, this continuous mutability in the electoral system has not only made the whole political-institutional system very weak, but also degraded it in its function in the eyes of the voters, as it seemed a clearly inefficient tool with respect to the needs of the constitutional system. Therefore, the present contribution aims to highlight the transformations that have taken place on the electoral system, trying to underline the diffculties of its consolidation, within the framework of the so-called Second Republic (1994-2020) and its new system of political parties.

RESUMEN: En los veintiséis años que caracterizan a las seis últimas legislaturas italianas (19942020) ha habido una intensa actividad politico-institucional, acompañada de una fuerte modificación del sistema de los partidos politicos. En este contexto, el sistema electoral y sus continuas modificaciones con nuevas leyes electorales ha influido fuertemente en la forma de gobierno, marcando su dinámica, tanto directa como indirectamente. Sin embargo, el rápido cambio de los diferentes sistemas electorales en los últimos años no ha estado acompañado de reformas parejas en el texto constitucional, produciéndose una asimetría en el funcionamiento de los nuevos sistemas electorales que se iban adoptando gradualmente, que los hacía sustancialmente incompletos, incoherentes y, en definitiva, frágiles. Todo ello ha terminado degradando el sistema a ojos de la ciudadanía. Las presentes notas tienen por objeto poner de relieve las transformaciones que se ban producido sobre el sistema electoral, tratando de subrayar las dificultades de su consolidación, en el marco de la llamada Segunda República (1994-2020) y de su nuevo sistema de partidos políticos.

KEY WORDS: electoral system, constitutional reform, political parties,

Palabras Clave: sistema electoral, reforma constitucional, partidos políticos

FECHA DE RECEPCIÓN: 19.01.2020

FeCHA DE ACEPTACIÓN: 19.02.2020 